\title{
Acute kidney injury, metabolic acidosis, and hypercalcemia with proximal tubular dysfunction-a diagnostic challenge: Questions
}

\author{
Adem Yasin Köksoy ${ }^{1}$ (D) \\ Received: 2 January 2021 / Accepted: 8 February 2021 / Published online: 17 March 2021 \\ (C) IPNA 2021
}

\section{Case summary}

A thirty-day-old male patient was admitted with the complaint of poor weight gain, anorexia, and somnolence. There was vomiting for 2 days but no diarrhea. He did not take any medications except vitamin D 400 IU/day. His past medical history revealed that he was admitted to the neonatal intensive care unit (NICU) for respiratory difficulty at birth and stayed at the hospital for 10 days. The pregnancy of the mother had been uncomplicated, and the family history was unremarkable. Parents were second-degree relatives. Physical examination revealed a male infant with body weight: $3.1 \mathrm{~kg}$ (SDS -1.91 ), height: $51 \mathrm{~cm}$ (SDS -1.09 ), and body mass index (BMI): $11.92 \mathrm{~kg} / \mathrm{m}^{2}$ (SDS -2.21). Infant was lethargic and dehydrated, and his skin was wrinkled.

Laboratory examination showed white blood cell (WBC): 9300/ $\mathrm{mm}^{3}$, hemoglobin (Hgb): $11.5 \mathrm{~g} / \mathrm{dl}$, platelet (Plt): 425.0007mm3, blood urea: $94 \mathrm{mg} / \mathrm{dl}$ (N 0-50), creatinine (Cre): $2.45 \mathrm{mg} / \mathrm{dl}(\mathrm{N} 0.1-1.2)$, sodium (Na): $135 \mathrm{mmol} / \mathrm{l}$, potassium $\left(\mathrm{K}^{+}\right): 2.2 \mathrm{mmol} / \mathrm{l}$, chloride $(\mathrm{Cl}): 103 \mathrm{mmol} / \mathrm{l}$, total protein: $6.1 \mathrm{mg} / \mathrm{dl}(\mathrm{N}: 6.4-8.3$ ), albumin (alb): $4.03 \mathrm{~g} / \mathrm{dl}$, calcium (Ca): $12.7 \mathrm{mg} / \mathrm{dl}(8.2-10.5)$, phosphorus $(\mathrm{P}): 5.98 \mathrm{mg} / \mathrm{dl}$ (N 3.5-6.6), magnesium (Mg): $1.8 \mathrm{mg} / \mathrm{dl}(\mathrm{N} 1.7-2.55)$, alanine amino transferase (ALT): $18.2 \mathrm{U} / 1$ (N 0-41), aspartate amino transferase (AST): $24 \mathrm{U} / 1$ (N 0-37), and uric acid (u.a.): $4.5 \mathrm{mg} / \mathrm{dl}$ (N 0-7.06). Blood gas analysis revealed metabolic acidosis ( $\mathrm{pH} 7.32, \mathrm{PCO}^{2} 34.8 \mathrm{mmHg}, \mathrm{HCO}_{3} 18.2 \mathrm{mmol} / \mathrm{l}$ ).

The answers to these questions can be found at https://doi.org/10.1007/ s00467-021-05033-8.

Adem Yasin Köksoy

ayasin71@gmail.com

1 Department of Pediatric Nephrology, Van Training and Research Hospital, University of Health Sciences, Süphan Mahallesi Hava Yolu Kavşağı 1. Kilometre Edremit, Van, Turkey
Urinalysis showed density $1006, \mathrm{pH} 6.5$, protein $1(+)$, and glucose $3(+)$ with no erythrocytes nor leucocytes. Spot urinary electrolytes and glucose at admission were as follows: $\mathrm{Ca} / \mathrm{Cre} 1.14 \mathrm{mg} / \mathrm{mg}$, u.a./Cre $1.1 \mathrm{mg} / \mathrm{mg}$, glucose/Cre 21.5 $\mathrm{mg} / \mathrm{mg}, \mathrm{Na} / \mathrm{Cre} 90 \mathrm{mmol} / \mathrm{mmol}, \mathrm{Cl} / \mathrm{Cre} 60.7 \mathrm{mmol} / \mathrm{mmol}$, $\mathrm{K} / \mathrm{Cre} 41.6 \mathrm{mmol} / \mathrm{mmol}$, and tubular reabsorption rate (TPR) $70 \%$.

Additional laboratory investigations revealed serum 25-OH vitamin D level $39.7 \mathrm{ng} / \mathrm{ml}(10-47)$, and parathormone (PTH) level was $9.6 \mathrm{pg} / \mathrm{ml}$ (15-65). Multiple crystalloids measuring $2.5 \mathrm{~mm}$ and $3 \mathrm{~mm}$ in both kidneys and accompanying medullary echogenicity were interpreted as medullary nephrocalcinosis (MNC) in abdominal ultrasonography.

Echocardiography showed ventricular septal defect. Patient was hospitalized. Appropriate fluid and electrolyte replacement was initiated. During follow-up, despite blood urea and creatinine being normalized, metabolic acidosis and glucosuria persisted until the 10th day. Further follow-up and investigations gave a definitive diagnosis.

\section{Questions}

1. What is your diagnosis?

2. What should be the diagnostic approach for metabolic acidosis?

3. What additional tests are needed for a definitive diagnosis?

4. How would you manage this patient?

\section{Declarations}

Conflict of interests The author declares no competing interests.

Publisher's note Springer Nature remains neutral with regard to jurisdictional claims in published maps and institutional affiliations. 